\title{
RBF Neural Network Control for USV with Input Saturation
}

\author{
Hua Deng, Renqiang Wang, Jingdong Li, Dawei Chen, Jianming Sun, Yue Zhao, Jiabao Du \\ College of Navigation, Jiangsu Maritime Institute, Nanjing 211170, PR China
}

\begin{abstract}
Intelligent control for USV with input saturation based on RBF network was proposed. Firstly, sliding surfaces with integral were designed on the basis of the sliding mode variable structure control technology. Secondly, RBF network was applied to approximate compensate the input saturation of system, and which was optimized by Genetic Algorithms. Finally, the control algorithm for USV was deduced by backstepping method with Lyapunov theory on the basis of sliding mode control. Relevant simulations show the control method is available for USV motion control.
\end{abstract}

\section{Introduction}

Unmanned Surface Vehicle (USV) due to the advantages of small size, mobility and other characteristic has become an important development trend of the future of the ship and which is mainly used to implement the dangerous task. So, it is playing increasing roles in commercial, scientific and military applications [1]. As the disturbances of wind, wave, current and other marine environment, the course of USV will inevitably deviate from the original given course when navigating at sea. While course control is a prerequisite to solve the trajectory tracking, autonomous navigation and collision avoidance and other issues. So, Steering control is one of the important research topics in the field of motion control [2] for USV.

At present, the motion control of most ships is on the basis of PID which has the advantages of simple algorithm structure and easy project realization, but its control parameters entirely rely on artificial experience judgments, therefore, it can only achieve limited intermittent adjustment with poor adaptive ability. A series of control theories and methods have been applied in USV heading control with the rapid development and progress of computer technology, such as Lyapunov stability theory and method [3], backstepping control algorithm [4,5], sliding mode control theory and technology [6-8], intelligent control theory and technology represented by fuzzy algorithm [9-12] and neural network [13-15].

In this paper, a kind of RBF network control algorithm for USV is proposed on the basis of Nomoto mathematical model and combination of integral backstepping control and sliding mode control.

\section{Problem description}

\subsection{USV plane motion model}

In actual straight-line voyages, ship movements usually exhibit a non-linear state. Therefore, in the mathematical model of plane motion, the nonlinear model for USV is described as following with influence of interference.

$$
\left\{\begin{array}{l}
\dot{x}_{1}=x_{2} \\
\dot{x}_{2}=f(x)+g \cdot u+d t \\
y=x_{1}
\end{array}\right.
$$

Where, $x_{1}$ and $x_{2}$ are the state variables of control system, $y$ is the actual course, $f(x)$ is the uncertain mathematical function of the system, and $f(x)=-1 / T H(\dot{\varphi}), H(\dot{\varphi})$ is unknown nonlinear mathematical function, $H(\dot{\varphi})=\dot{\varphi}+\alpha \dot{\varphi}^{3}, \varphi$ is actual course too, $\alpha$ is the constant to be determined. $g$ is the gain of control input, and $g=K / T \cdot u$ is control input representing rudder angle. $d t$ is external interference. $K$ is the index of ship's turning performance and $T$ is the index of ship's followability. These parameters can be obtained through ship handling experiment.

\subsection{Anti-saturation compensation based on RBF network}

It is well known that the USV is generally drived by rudder servo system. Thus, the actual characteristics of the rudder servo system, in general, the actual output of the rudder angle is less than $35^{\circ}$, must be taken into account in actual navigation with large-scale motor. To solve this problem, in this paper, the anti-saturation theory is cited first, and an online approximation and effective compensation is achieved by RBF neural network. 
Anti-saturation compensation for rudder servo system is described as follows, the maximum control input value is introduced as $u_{\max }$, and maximum value is $35^{\circ}$. In addition, it is constructed that $\delta=u-v$ and $u=\operatorname{sat}(v)$. Therefore, the control input restricted mathematical formula $\operatorname{sat}(v)$ was shown in equation (2).

$$
\operatorname{sat}(v)=\left\{\begin{array}{cc}
u_{\max } & v>u_{\max } \\
v & |v| \leq u_{\max } \\
-u_{\max } & v<-u_{\max }
\end{array}\right.
$$

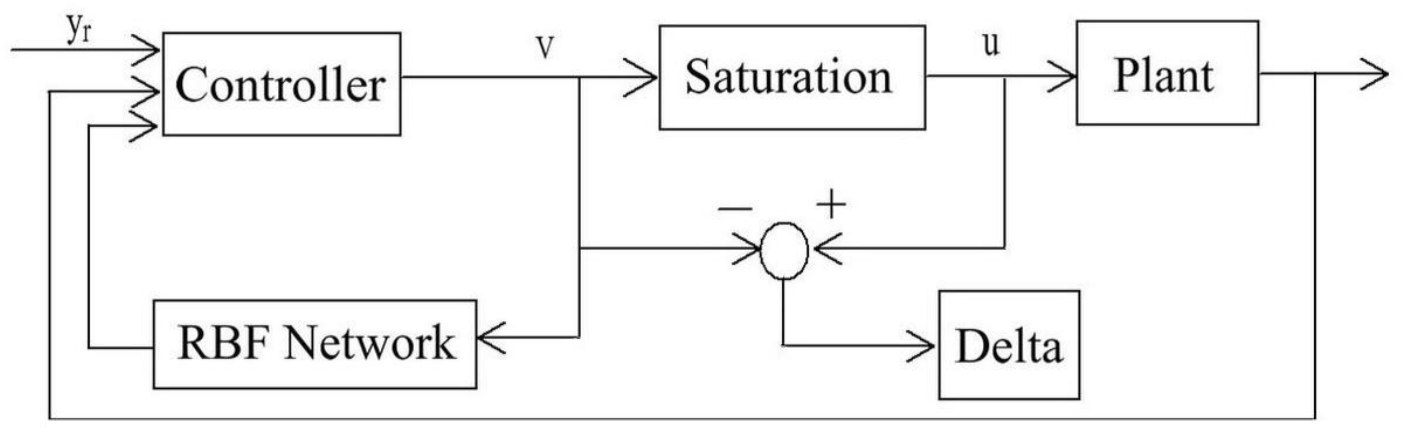

Fig. 1. Closed-loop control system with saturation

In paper, the RBF neural network is designed to approximate rudder output $\delta$ so as to realize effective compensation, where closed-loop control system diagram for USV is shown in figure 1 on the basis of RBF neural network compensation.

It is described that the mathematical model of RBF network is shown in equation (3).

$$
\left\{\begin{array}{l}
h_{j}=\exp \left(\frac{\left\|\mathbf{x}-\mathbf{c}_{j}\right\|^{2}}{2 b_{j}^{2}}\right) \\
\delta=\mathbf{W}^{* T} \cdot \mathbf{h}(\mathbf{x})+\varepsilon
\end{array}\right.
$$

Where, $\mathbf{x}$ is the input of RBF network, $j$ is a hidden layer of the network the first network input, $\mathbf{h}=\left[h_{j}\right]^{T}$ is high Gaussian function, $\mathbf{W}^{*}$ is the ideal for weighting value, $\varepsilon$ is ideal for neural network approximation error, and $\varepsilon \leq \varepsilon_{\max }$.

According to figure 1, the RBF network input is $x=v$, the network output $\hat{\delta}$ is obtained by equation (4).

$$
\left\{\begin{array}{l}
\hat{\delta}=\hat{\mathbf{W}}^{\mathbf{T}} \mathbf{h}(\mathbf{x}) \\
\tilde{\delta}=\delta-\hat{\delta}=-\tilde{\mathbf{W}}^{\mathbf{T}} \mathbf{h}(\mathbf{x})+\varepsilon
\end{array}\right.
$$

Where, $\tilde{\mathbf{W}}=\hat{\mathbf{W}}-\mathbf{W}^{*}$.

\subsection{RBF Neural Network Optimization}

RBF neural network has a universal property of approximation [16], while its ability of approximation depends entirely on network performance parameters, i.e., output weights $w_{i}$, width $b_{i}$ and the number of hidden center units $c_{i}$. However, it is very difficult to determine these parameters in advance. In view of that, GA is uesd to optimize RBF neural network, which is able to approach the internal uncertainties of ship model and external disturbance, so as to achieve the best performance of approaching. The process of RBF neural network parameters optimization is shown in Figure 2.

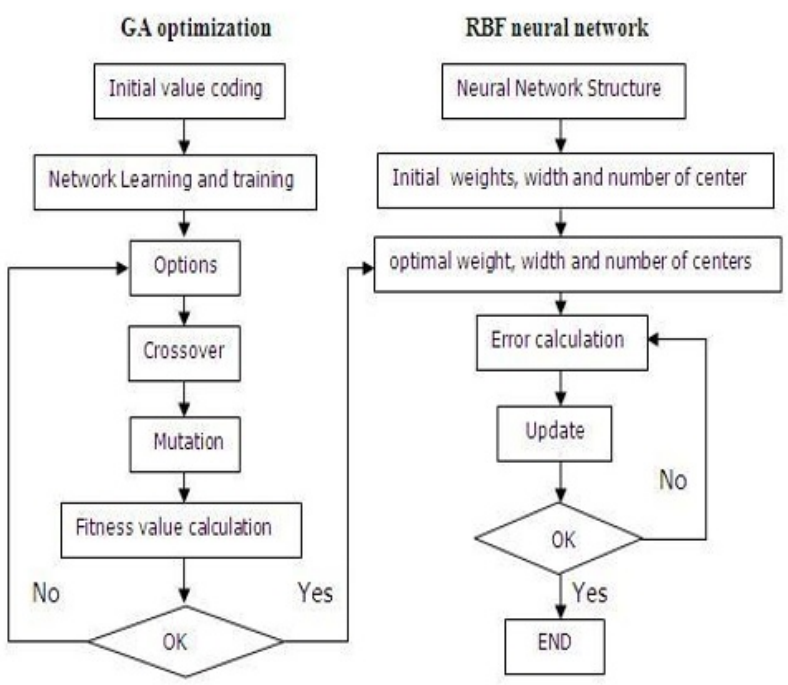

Fig. 2. RBF neural network optimization with GA

\section{Intelligent Steering control design based on backstepping}

The designing process of control law contains the following steps.

Firstly, according to the characteristics of the system (1), the sliding surfaces are defined as following:

$$
\left\{\begin{array}{l}
\dot{\xi}=z_{2} \\
z_{1}=x_{1}-\varphi_{r} \\
z_{2}=x_{2}-\sigma_{1}
\end{array}\right.
$$

Where, $\varphi_{r}$ is expected course, $\sigma_{1}$ and $\sigma_{2}$ are virtual stabilization function, $z_{1}$ is heading error, $\xi$ is the integral term which is added in order to eliminate the static error. 
Secondly, constructing the Lyapunov function to determine the asymptotic stability of control system.

According to equation (5), it is obtained equation (6).

$$
\dot{z}_{1}=z_{2}+\sigma_{1}-\dot{\varphi}_{r}
$$

The virtual stabilization $\sigma_{1}$ is set as equation (7)

$$
\sigma_{1}=-k_{1} z_{1}+\dot{\varphi}_{r}
$$

Therefore, it can be obtained equation (8)

$$
\dot{\sigma}_{1}=-k_{1}\left(-k_{1} z_{1}+z_{2}\right)+\ddot{\varphi}_{r}
$$

Similarly, it is obtained equation (9) and equation (10) according to equation (5).

$$
\begin{gathered}
\dot{z}_{1}=-k_{1} z_{1}+z_{2} \\
\dot{z}_{2}=\dot{x}_{2}-\dot{\sigma}_{1}=f(x)+g u+d t-\dot{\sigma}_{1}
\end{gathered}
$$

And, the first Lyapunov function is constructed as equation (11).

$$
V_{1}=\frac{1}{2} z_{1}^{2}+\frac{1}{2} z_{2}^{2}
$$

It can be inferred equation (12) by equation (11).

$$
\dot{V}_{2}=-k_{1} z_{1}^{2}+z_{1} z_{2}+z_{2}\left[f(x)+g u+d t-\dot{\sigma}_{1}\right]
$$

The second Lyapunov function is constructed as equation (13).

$$
V_{3}=V_{2}+\frac{\lambda}{2} \xi^{2}
$$

Similarly, it can be inferred equation (14) by equation (13).

$$
\begin{aligned}
\dot{V}_{3} & =\dot{V}_{2}+\lambda \xi \dot{\xi} \\
& =-k_{1} z_{1}^{2}+z_{2}\left[z_{1}+f(x)+g u+d t-\dot{\sigma}_{1}+\lambda \xi\right]
\end{aligned}
$$

Thirdly, according to figure 2 , it is known that $u=v+\delta$, which is taken into equation (14). Therefore, it can be obtainedequation (15).

$$
\dot{V}_{3}=-k_{1} z_{1}^{2}+z_{2}\left[z_{1}+f(x)+g(v+\delta)+d t-\dot{\sigma}_{1}+\lambda \xi\right]
$$

The following control law with damping law [9] is designed by the RBF network.

$$
v=\frac{1}{g}\left[-z_{1}-f(x)-\left(k_{2}+\eta\right) z_{2}+\dot{\sigma}_{1}-\lambda \xi\right]-\hat{\delta}
$$

It can be inferred equation (17), according to equation (15) and equation (16)

$$
\dot{V}_{3}=-k_{1} z_{1}^{2}-k_{2} z_{2}^{2}-\eta z_{2}^{2}+z_{2} g \tilde{\delta}+z_{2} d t
$$

Finally, the total Lyapunov function is constructed as equation (18).

$$
L=V_{3}+\frac{1}{2} \gamma \tilde{\mathbf{W}}^{T} \tilde{\mathbf{W}}
$$

Where, $\gamma>0$.

Therefore, it can be inferred equation (19) by equation (18)

$$
\begin{aligned}
\dot{L} & =-k_{1} z_{1}^{2}-k_{2} z_{2}^{2}-\eta z_{2}^{2}+z_{2} g \tilde{\delta}+z_{2} d t+\gamma \tilde{W}^{T} \dot{\hat{W}} \\
& =-k_{1} z_{1}^{2}-k_{2} z_{2}^{2}-\eta z_{2}^{2}+\tilde{W}^{T}\left(\gamma \dot{\hat{W}}-\mathbf{h} z_{2}\right)+[d(t)+\varepsilon+g \tilde{\delta}] z_{2}
\end{aligned}
$$

In order to ensure system asymptotically stable, the following adaptive law is designed.

$$
\dot{\hat{W}}=\frac{1}{\gamma} \mathbf{h} z_{2}
$$

It is easy obtained formula (21) by theorem “ $x y \leq \eta x^{2}+\frac{1}{4 \eta} y^{2}$ ".

$$
\varpi z_{2} \leq \eta z_{2}^{2}+\frac{1}{4 \eta}\|\varpi\|_{\infty}^{2}
$$

Where, $\varpi=d(t)+\varepsilon+g \tilde{\delta}$.

Therefore, the formula (22) can be acquired .

$$
\begin{aligned}
\dot{L} & =-k_{1} z_{1}^{2}-k_{2} z_{2}^{2}-\eta z_{2}^{2}+\varpi z_{2} \\
& \leq-k_{1} z_{1}^{2}{ }_{1}-k_{2} z_{2}^{2}+\frac{1}{4 \eta}\|\varpi\|_{\infty}^{2} \\
& \leq-k_{2} z_{2}^{2}+\frac{1}{4 \eta}\|\varpi\|_{\infty}^{2}
\end{aligned}
$$

In order to ensure that the system (1) is asymptotically stable, it is only need to prove the establishment which is $k_{2} z_{2}^{2} \geq \frac{1}{4 \eta}\|\omega\|_{\infty}^{2}$. Proof slightly, see the literature [4].

So, the entire control system is asymptotically stable.

\section{Simulation}

\subsection{Simulated USV model}

The USV "Lanxin" was adopted in simulation, and the parameters of planar motion mathematical model were listed as table 1. So, the parameters of Nomoto model of "Lanxin" were $K=1.502, T=0.905, \alpha_{1}=0.001$, $\alpha_{i(i=1,2,3 \ldots)}=0$, when its speed is 8.5 knots.

Table 1. The "LanXin" USV Parameters

\begin{tabular}{c|c}
\hline Length Between Perpendiculars & $7.02 \mathrm{~m}$ \\
\hline Breadth & $2.60 \mathrm{~m}$ \\
\hline Speed & $<=35 \mathrm{kn}$ \\
\hline Draft (full load) & $0.32 \mathrm{~m}$ \\
\hline Block Coefficient & 0.6976 \\
\hline Displacement (full load) & $2.73 \mathrm{~m}^{3}$ \\
\hline Rudder Area & $0.2091 \mathrm{~m}^{2}$ \\
\hline $\begin{array}{c}\text { Distance Between Barycenter } \\
\text { and Center }\end{array}$ & $0.35 \mathrm{~m}$ \\
\hline
\end{tabular}

At the same time, the model of rudder servo system which was described as equation (23) was added into nonlinear Nomoto model of "Lanxin".

$$
\dot{\delta}=-\frac{1}{T_{r}} \delta+\frac{1}{T_{r}} \delta_{r}
$$

Where, $T_{r}$ is time constant, and the value of which is generally about 0.2 seconds. $\delta_{r}$ is executed rudder angle. $\delta$ is real-time rudder angle, and the restriction of which was shown in equation (24). 


$$
\delta=\left\{\begin{array}{cc}
35^{\circ} & \delta>35^{\circ} \\
\delta & |\delta| \leq 35^{\circ} \\
-35^{\circ} & \delta<-35^{\circ}
\end{array}\right.
$$

In addition, it is considered that ship's movement is greatly affected by wind and wave. And the model can be replaced by a transfer function of second-order wave and a white noise [17].

\subsection{Condition setting}

The ideal angle command is $x_{d}=\sin (t)$. In order to show that the control system compensates the control input limited capacity, a larger initial error is used, and the initial vector of the system is $\left[\begin{array}{ll}10 & 0\end{array}\right]$. RBF network structure is adopted as figure 1, and network input is $x=v$, according to the actual input range of the network to design Gaussian function of the parameters, taking $C_{i}=\left[\begin{array}{lllll}-1.0 & -0.5 & 0 & 0.5 & 1.0\end{array}\right]$ and $b_{i}=5$, the network initial value is zero. Using the control law equation (19) and adaptive law equation (24), where $c=5, \gamma=10$. In the sliding mode control, the saturation function is used instead of the switching function, and the boundary layer thickness $\Delta$ is 0.02 .

\subsection{Simulation and analysis}

Simulation of USV course tracking control was carried out on the basis of Matlab/Simulink platform, and the results were shown in figure 3, figure4 and figure5.

The output response of angle tracking and speed tracking of USV was depictted in figure 3. And from two pictures, it can be seen that course angle reaches the set value with no overshoot.

And the output response of restricted front and rear control inputs was described in figure 4 . And from this picture, it can be seen that an internal auxiliary compensation mechanism designed with anti-saturation control technique can make control input quickly quit saturation.

And also the output response of rudder angle and approximation was bewrited in figure 5. And from this picture, it can be seen that an internal auxiliary compensation mechanism on the basis of RBF network has effective online approximation, which is proposed by anti-saturation control technique.
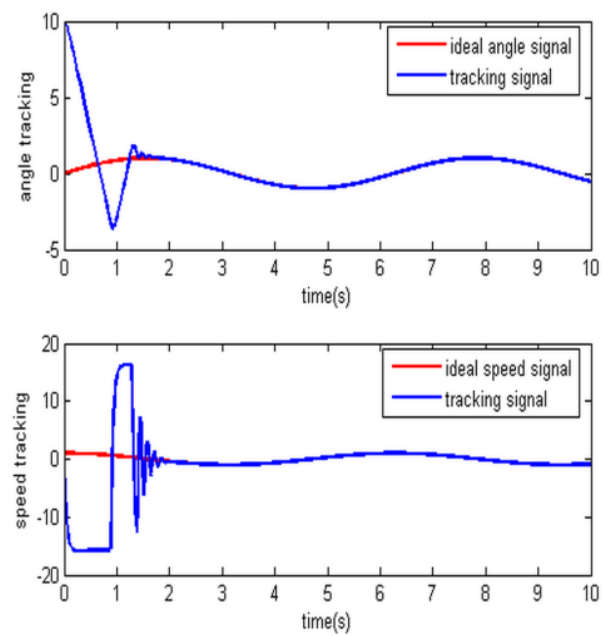

Fig. 3. Position and speed tracking.
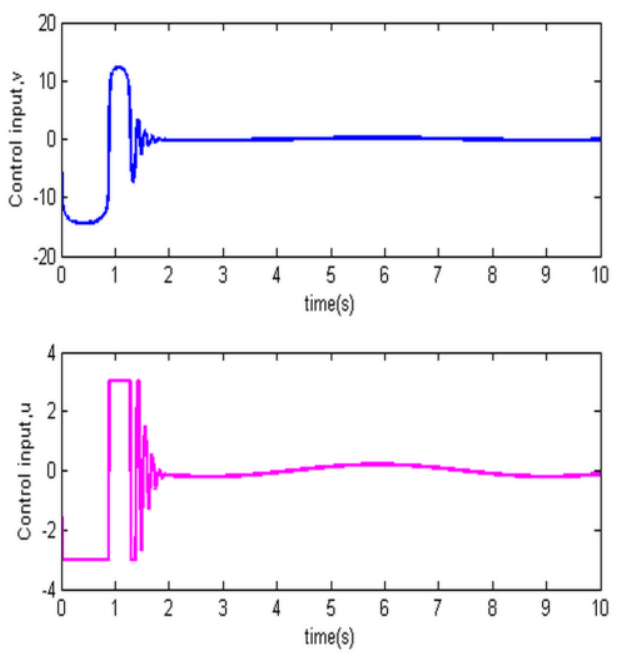

Fig. 4. Restricted front and rear control inputs $v$ and $u$.

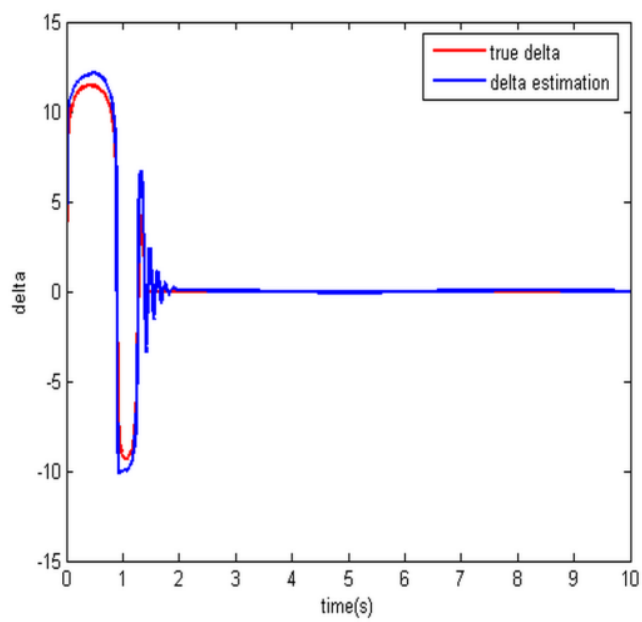

Fig. 5. Controls the input limit value $\delta$ and the approximation. 


\section{Conclusion}

Intelligent steering control for USV with input saturation was proposed based on RBF network. It is shown that an internal auxiliary compensation mechanism designed with anti-saturation control technique can make control input quickly quit saturation, and RBF neural network optimized by GA has effective online approximation. Therefore, the intelligent control algorithm designed in this paper is available for USV.

\section{Acknowledgment}

This work was supported by Natural Science Research Project of Universities in Jiangsu Province under Grant No.18KJB580003, the Foundation of Jiangsu Maritime Institute under Grant No.XR1501, and Top-notch Academic Programs of Jiangsu Higher Education Institutions under Grant No. PPZY2015B177.

\section{References}

1. S. Bennett, Nicolas Minorsky and the automatic steering of ships, IEEE Control Systems Magazine, 4, 1984, 10-15.

2. S. Kumarawadu, K. Kumara, On the speed control for automated surface vessel operation, in Proceedings of the 2007 third international conference on information and automation for sustainability, Melbourne, Australia, 2007, 135-140.

3. B. Volker, Unmanned Surface Vehicles - A Survey, Skibsteknisk Selskab, Copenhagen, Denmark, 2008.

4. Benaskeur A. R, Desbines A, Backstepping based adaptive PID contro, Proceedings of Control Theory and Applications, 149, 2002, 54-59.

5. Y. Pan, D. Huang, Z. Sun, Backstepping adaptive fuzzy control for track-keeping of under-actuated surface vessels, Control Theory and Applications, 7, 2011, 907-914.

6. Wang Renqiang, Gong Jianyun, Zhao Yuelin, Neural Network Sliding Mode Control under New Reaching Law and Application, Proceedings of Advanced Information Technology, Electronic and Automation Control Conference, 12, 2015, 911-917.

7. Renqiang Wang, Yuelin Zhao, Keyin Miao, Application of Neural Network Minimum Parameter Learning Algorithm in Ship's Heading Tracking Control, Proceedings of 9th International Symposium on Computational Intelligence and Design, 12, 2016, 135-139.

8. K. Do, Z. Jiang, J. Pan, Under-actuated ship global tracking under relaxed conditions, IEEE Trans. on Automatic Control, 47, 2002, 1592 - 1536.

9. Wang Ning, Lv Shuailin, Liu Zhongzhong, Global finite-time heading control of surface vehicles, Neurocomputing, 175, 2016, 662-666.

10. X. Bao, K. Nonami, Z. Yu, Combined yaw and roll control of an autonomous boat, Proceedings of the
2009 IEEE International Conference on Robotics and Automation, Kobe, Japan, 2009, 188-193.

11. T. Li, Y. Yang, B. Hon, Robust adaptive fuzzy design for ships track-keeping control, Control Theory and Applications, 03, 2007, 445-448.

12. L. Yuan, H. Wu, Multiple sliding mode adaptive fuzzy controller for nonlinear marine autopilot systems, CAAI Transactions on Intelligent Systems, 04, 2010, 308-312.

13. Guanshan $\mathrm{Hu}$, Yingbing Zhou, Application of Fuzzy Neural Network in Ship Course Control, Applied Mechanics and Materials, 1511, 2012, 309-315.

14. Xin Wang, Tieshan Li, Weilin Luo, Direct Adaptive Neural Network Control for a Class of Ship Course Uncertain Discrete-time Nonlinear Systems, Marine Engineering Frontiers, 37, 2013, 42-48.

15. Yang Wang, Chen Guo, Fuchun Sun, Dynamic neural fuzzified adaptive control of ship course with parametric modelling uncertainties, Int. J. of Modelling, Identification and Control, 13, 2015, 251258.

16. Wang Renqiang, Zhao Yuelin, Sun Jianming, Application of Optimized RBF Neural Network in Ship's Autopilot Design, Proceedings of IEEE Advanced Information Management, Communicates, Electronic and Automation Control Conference, 10, 2016, 1642-1646.

17. FAN Yunsheng, SUN Xiaojie, WANG Guofeng, GUO Chen, On Fuzzy Self-adaptive PID Control for USV Course, Proceedings of the 34th Chinese Control Conference, 7, 2015, 8472-8478. 